This item was submitted to Loughborough's Research Repository by the author.

Items in Figshare are protected by copyright, with all rights reserved, unless otherwise indicated.

\title{
Multiphonon absorption of light in nonpolar crystals
}

PLEASE CITE THE PUBLISHED VERSION

PUBLISHER

(C) American Physical Society

LICENCE

CC BY-NC-ND 4.0

REPOSITORY RECORD

Kusmartsev, F.V.. 2019. "Multiphonon Absorption of Light in Nonpolar Crystals". figshare.

https://hdl.handle.net/2134/1302. 


\title{
Multiphonon absorption of light in nonpolar crystals
}

\author{
F. V. Kusmartsev \\ Institut fur Theoretische Physik, Universität zu Köln, D-5000 Köln 41, Germany \\ and Lev Davidovich Landau Institute for Theoretical Physics, GSP-1, 117940, Moscow, V-334, U.S.S.R. \\ (Received 11 January 1990; revised manuscript received 22 May 1990)
}

\begin{abstract}
We further develop the theory of Urbach tails (Urbach rule). The presented theory of the light absorption below the band edge is based on the assumption that the light absorption below the band edge is due to the tunnel-activated transitions of the lattice. Each type of tunnel-activational transitions corresponds to a class of multiphonon instantons, which is associated with the regime of light absorption. The theory presented predicts the existence of certain singularities in the Urbach tail. We propose an experiment to detect these kinds of singularities. We have made calculations on the model of Frenkel excitons interacting with nonpolar deformational optical phonons in threedimensional crystals and determined the "phase diagram" of different regimes of light absorption. The possibility of experimental studies of these diagrams in the Urbach tail is discussed.
\end{abstract}

\section{INTRODUCTION}

We develop a systematic theory of the absorption of light below the band edge. The approach is based on the assumption that many phonons participate in the absorption process by means of tunnel-activated transitions of the lattice. ${ }^{1}$ There are two major directions which may be followed in the theory of the band-edge light absorption. In the first one the absorption is due to static inhomogeneities of the potential. ${ }^{2}$ In the second direction the dispersion of the band edge is due to thermal oscillations of the lattice (see Ref. 1 and references contained therein).

Here we construct and classify the optimal solutions of the model describing a Frenkel exciton that interacts with deformational optical phonons of a frequency $\omega$. We neglect the influence of the acoustic phonons on the light absorption, since we consider the case when the deficiency of the photon energy $\Delta$ is much larger than the phonon energy $\hbar \omega$, where $\Delta=E_{g}-\hbar \Omega$ is the difference between the energy gap $E_{g}$ and the photon energy $\hbar \Omega$. In this case we may adopt the quasiclassical approach considering the light absorption below the band edge as a tunnel motion of the lattice in a potential, including an adiabatic potential. Using imaginary time variables the process may be described as a classical motion in a constructed potential.

The model investigated has been considered before in Refs. 3 and 4 . In Ref. 3 the rate of self-trapping of electrons or excitons has been estimated (about the theory of the self-trapping see details in Ref. 5). In Ref. 4 two mechanisms of the light absorption have been explored and several asymptotic solutions have been derived. However, they do not give a complete picture of light absorption. Moreover, in Ref. 4 incorrect exclusions of phonon variables have been made. On the other hand, in our approach we exclude exciton variables and obtain an effective action, which depends only on the phonon variables. In such an approach we may construct a complete classification of a complete set of all classical solutions in imaginary time (instantons), considering directly the tunnel motion of the lattice in a potential, which includes an adiabatic potential.

Our study of the model considered employs two methods: the scale substitution method ${ }^{6}$ and the method of a weakly bound state of the exciton. ${ }^{7}$ In the first case we have obtained an exactly solvable model. In the second case we have obtained the one-mode model with curvature, which depends on the deficiency energy $\Delta$ of the photon and on the temperature $T$. The results obtained are presented in the form of suitable $\Delta, T$ phase diagrams. Each region in such a diagram is characterized by an appropriate optimal way of the tunnel transition.

\section{CLASSIFICATION OF THE ABSORPTION PROCESSES}

Since we are only interested in the exponential dependencies of the absorption coefficient on the photon frequency and the temperature we may employ the FranckCondon principle in conjunction with a simple mechanical description of the lattice motion. We describe the lattice motion by the configuration coordinates of the deformation, corresponding to the optical deformational phonons. From the quasiclassical viewpoint the absorption of light below the edge of the exciton band must be treated as an optimal transition between different branches of the lattice potential.

For the classification of instantons it is convenient to introduce the three different energy branches $E, B$, and $F$ (see, for details, Ref. 1). The bound (free) branch $B(F)$ corresponds to the energy of the lattice and the exciton in the bound (free) state. The empty $E$ branch corresponds to the sum of the pure elastic energy of the lattice and the photon energy. According to the Franck-Condon principle the system conserves its configuration coordinates during the electronic transition of the photon absorption. Since the absorption may only occur into the bound- 
exciton state, the transition of the lattice from the $E$ branch to the $B$ branch (it corresponds to the moment of the generation of the exciton) occurs in some configuration of the lattice in which the bound energy of the exciton is exactly equal to the photon energy deficiency. The deformation of the lattice in this excitonic state is not equal to the optimal deformation of the lattice at a given temperature (thermal oscillation of the lattice). Therefore the lattice tunnels over the $E$ branch from the optimal thermal deformation to the deformation in which the bound energy of the exciton with the lattice is equal to the photon energy deficiency.

Each classical solution in imaginary time corresponds to the way of tunnel motion of the lattice over these energy branches and depends on their configuration. The configuration of the energy branches at some arbitrarily chosen deficiency value is presented in Fig. 1. The horizontal lines (the lines of constant energy $\varepsilon$ ) correspond to the lattice motion at different temperatures and to the $E B F$ ( $E B$ in the self-trapped channel), $E B, B$, and $A$ trajectories, respectively. The point $i$ on the horizontal line $E B F-E B$ is the initial point of the tunnel motion. The point $f$ on the horizontal line $E B F-E B$ is the final point of the tunnel motion.

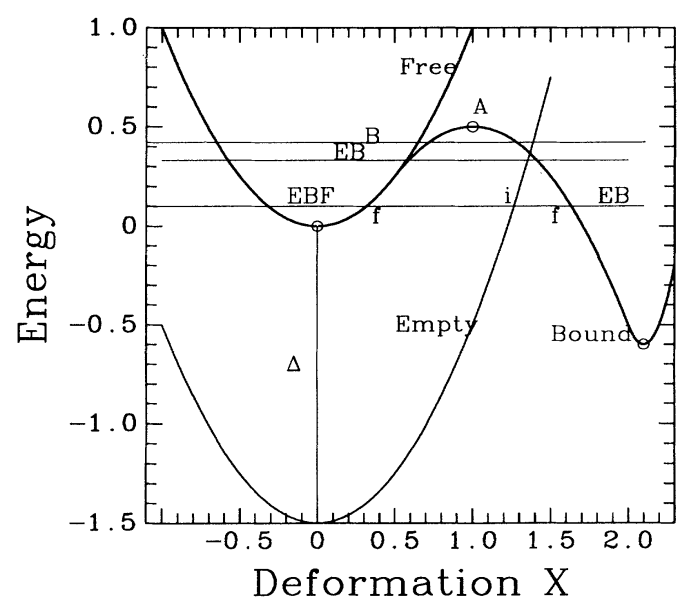

FIG. 1. The configuration of energy branches (empty, bound, and free). The empty branch is the elastic energy of the lattice and the photon energy. The free branch is the elastic energy of the lattice and the energy of the free exciton. The circle on this branch $F$ corresponds to a free exciton at zero temperature of the lattice. The bound branch is the elastic energy of the lattice and the energy of the exciton in a bound state with the lattice. The circle on this branch $B$ corresponds to a self-trapped exciton at zero temperature of the lattice. The value of the photon energy deficiency $\Delta=E_{g}-\hbar \Omega$ is chosen arbitrarily. The horizontal lines correspond to the energy parameter $\varepsilon$, which characterizes the lattice temperature, and to the different instanton solutions: $E B F, E B$, and $B$, respectively. The point $i$ is the initial point of the tunnel motion. The point $f$ is the final point of the tunnel motion. The circle $A$ corresponds to the top of the self-trapped barrier and the activational instanton.
We may distinguish two different types and eight classes of tunnel ways. Each type may involve several classes. A type depends on the final state of the exciton in the tunnel transition considered. Each type of instanton defines a particular channel of the light absorption. As a result of the tunnel transition of the lattice the exciton can be either free (lattice on the free branch) or selftrapped (lattice on the bound branch $B$, see Fig. 1). Therefore there are two types of phonon trajectories connected with two channels of light absorption: free and self-trapped. Each class depends on the way of the tunnel motion of the lattice, from the state without an exciton into a state with an exciton. Each way depends on the number of energy branches and the order of the tunnel motion over these energy branches $E, B$, and $F$.

In the free channel there are the following tunnel ways.

(1) The first part of the motion is the tunnel motion of a lattice without an exciton ( $E$ motion), the second part of the motion is the tunnel motion in the presence of an exciton in a bound state ( $B$ motion), and the last third part of the motion is the tunnel motion in the presence of an exciton in a free state ( $F$ motion); this class of the absorption processes corresponds to the horizontal line in Fig. 1, designated $E B F$. The point $i(f)$ corresponds to the initial (final) point of the tunnel motion.

(2) Only $E$ and $B$ motion; this way corresponds to the horizontal line in Fig. 1, which is designated as $E B$.

(3) Only $B$ and $F$ motion; this way exists at the configuration of the energy branches corresponding to the large values of the deficiency, where the level of the intersection of empty and bound branches is below the level of the coalescence of the bound and free branches.

(4) Only $B$ motion; this class of ways corresponds to the horizontal line in Fig. 1, which is designated as $B$. In this case the motion in the free energy branch is the classical one (in real time) and therefore does not give a contribution to the imaginary part of the action.

(5) Activational solution $(A)$, the lattice does not move during the time of the tunneling. This solution corresponds to the circle on the top of the self-trapped barrier. In this solution all rearrangements of the lattice exist in the classically permitted region. And there are no rearrangements in the classically forbidden region. For this reason this solution occurs only at the high temperature, when the tunnel time is very small. The physical interpretation of this solution is simple: it is an activational jump of the lattice through the self-trapped barrier. This class of ways at vanishing photon energy deficiency in the self-trapped channel coincides with the activational solution obtained in the theory of self-trapping. ${ }^{3-5}$

In the self-trapped channel there are only three classes of instantons: the second, fourth, and fifth class discussed above. The form of different classes of trajectories is presented in Fig. 2. Each trajectory is presented in the plane of configuration coordinates of the lattice deformation $X$ and of the imaginary time axis (IT). The first ( $A$ instanton) is a static one. These activational trajectories have been taken into account in Refs. 8-11. The other trajectories of the forms presented in Fig. 2(a), missing in Refs. 8-11, may be considered in the framework of the formalism of Ref. 4. It is very interesting to note that 
several trajectories [see Fig. 2(b)] have an inverse part of the motion. The parts of trajectories corresponding to the motion over $E$ and $B$ branches create a cusp [see Fig. 2(b)]. Such respective trajectories are very important for the light absorption in the free channel at small values of the photon energy deficiency $\hbar \omega<\Delta<W$, where $W$ is the value of the self-trapped barrier (see Fig. 1). In this region of the deficiency the light absorption in the selftrapped channel is negligible in comparison with the free channel and approximately coincides with the rate of the exciton self-trapping. In this case the light absorption is
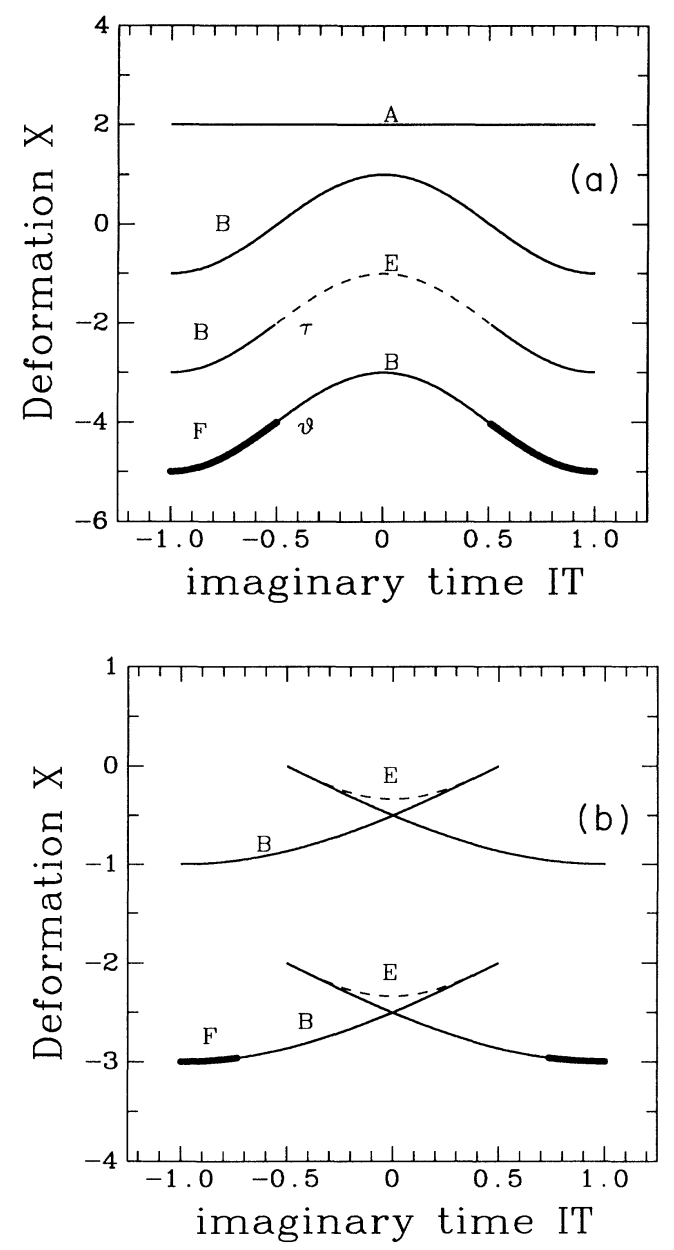

FIG. 2. (a) The shapes of different trajectories, which correspond to $A, B, E B$, and $B F$ instantons, respectively. The letters $B, E$, and $F$ correspond to the motion over $B$ (solid line), $E$ (dashed line) and $F$ (thick solid line) energy branches, respectively. The letter $\tau$ gives the time of the transition from the $E$ to the $B$ energy branch. The letter $\vartheta$ corresponds to the time of the transition from the $B$ to the $F$ energy branch. (b) The shapes of different respective trajectories, which correspond to $E B$ and $E B F$ instantons for the free channel, respectively. The letters $E$, $B$, and $F$ correspond to the motion over the $E, B$, and $F$ energy branches, respectively. mainly due to the tunnel motion over these respective trajectories, which have not been considered in the earlier work.

At each value of the deficiency energy $\Delta$ and the temperature $T$ only one of these classes is optimal, i.e., it corresponds to the minimal value of the action. Each optimal class of instantons at a given value of the deficiency energy $\Delta$ and the temperature $T$ defines the regime of the light absorption. Thus, in a self-trapped channel, there are three regions [in the $(\Delta, T)$ plane], corresponding to the three regimes of the light absorption: $E B, B$, and $A$. The transition from one region to another one by changing the temperature $T$ and the deficiency energy $\Delta$ can occur in two different manners. In the first of them there is a singularity in the dependence of the coefficient of the absorption of light on the deficiency energy or the temperature (the abrupt changing of the slope). In this case we speak of a first-order transition. In the second case this dependence is smooth. We call the smooth transition a transition of second order. In the first case there exists the abrupt changing of the form of trajectories from one class to another one. In the second case we have a smooth changing of the form of trajectories from one class to another one.

\section{THE SELF-SIMILAR ANSATZ}

Next, we calculate the exponential dependence of the coefficient of light absorption on the photon energy deficiency $\Delta$ and the temperature $T$. We follow the formalism, which is based on the quasiclassical approximation and Franck-Condon's principle.

The absorption coefficient of light at the band edge is defined by ${ }^{1}$

$$
K(\Delta) \approx \exp \left[i \hbar^{-1} S_{0}(\Delta, \beta)\right]
$$

where we get for the quasiclassical action

$$
S_{0}(\Delta, \beta)=\int_{-i \hbar \beta / 2}^{i \hbar \beta / 2} L\left(X, X^{\prime}\right) d t
$$

Here, the parameter $\beta$ is an inverse temperature $\beta=\omega / T$ (we use units in which $\hbar=1$ ), $L\left(X, X^{\prime}\right)$ is the Lagrange function of the lattice describing the motion over the $E, B$, and $F$ branches, and $X(R)$ are the phonon variables. It is convenient to introduce the dimensionless units

$$
r \rightarrow 2 m E_{\mathrm{FC}} v r, \quad t \rightarrow t / \omega, \quad X \rightarrow X / 2 m^{3 / 2} E_{\mathrm{FC}} v \omega
$$

and

$$
S_{0} \rightarrow \sigma_{0} S
$$

where

$$
\sigma_{0}=W / \omega, \quad W=1 / 4 m^{3} E_{\mathrm{FC}}^{2} v^{2}, \quad E_{\mathrm{FC}}=\omega \gamma^{2} .
$$

The parameter $m$ is the effective mass of the Frenkel exciton, $E_{\mathrm{FC}}$ is the Franck-Condon energy, the constant $\gamma$ is the constant of the interaction with deformational optical phonons, and $v$ is the volume per atom. In these units the deficiency of the photon energy $\Delta$ is measured by the value $W$ of the self-trapped barrier ${ }^{3-5}(W>\omega)$ and the action $S$ may be written 


$$
\begin{aligned}
S(\Delta, \beta)= & \int_{\Gamma}\left[-\Psi \frac{\Delta \Psi}{2}+\Psi^{2} X\right] d t d^{3} r \\
& +\int_{-i \hbar \beta / 2}^{i \hbar \beta / 2} \frac{\left(\dot{X}^{2}-X^{2}\right)}{2} d t d^{3} r
\end{aligned}
$$

Here function $\Psi$ is the wave function of the exciton, the path $\Gamma$ is the time contour, which will be suitably defined below for the different classes of trajectories.

Let us adopt a scale substitution, which, in imaginary time, has the form

$$
\begin{aligned}
& \Psi(r, t) \rightarrow \varphi^{3}(t) \Psi\left(\varphi^{2} r\right), \\
& X(R, t) \rightarrow \varphi^{4}(t) \Psi\left(\varphi^{2} r\right) .
\end{aligned}
$$

Function $\Psi(r)$ is the solution of the following equation:

$$
-\Delta \Psi / 2-\Psi^{3}=E \Psi
$$

where $E \equiv-C_{0} / 2=-44.4$ and

$$
X(r)=-\Psi^{2}(r) .
$$

As a result of this substitution we derive for the action

$$
S(\beta, \Delta)=C_{0}\left(\int_{-\beta / 2}^{\beta / 2}\left(\dot{\varphi}^{2}+\varphi^{2}\right) d t-\int_{\Gamma} \varphi^{4} d t\right) .
$$

Thus, with the aid of this substitution, we have reduced the original problem of a four-dimensional tunnel transition of the lattice to the description of a tunnel transition between two one-dimensional energy branches: (1) empty $E$ branch with a Lagrangian:

$$
L_{e}=C_{0}\left(\dot{\varphi}^{2}+\varphi^{2}\right) \text {, }
$$

and (2) bound $B$-branch with a Lagrangian:

$$
L_{b}=C_{0}\left(\dot{\varphi}^{2}+\varphi^{2}-\varphi^{4}\right)+C_{0} \Delta .
$$

The details of this reduction will be published elsewhere. ${ }^{6}$

\section{INSTANTONS AND REGIMES OF LIGHT ABSORPTION}

One can obtain a free exciton only in the free channel of light absorption and the self-trapped exciton only in the self-trapped channel of the light absorption. The exactly solvable model considered here has three classes of instantons in a free channel. They correspond to the three regimes of the light absorption.

(1) $E B$ regime; at first the tunnel motion follows the $E$ branch, then the $B$ branch (see line $E B$ in Fig. 1). The form of trajectory is presented in Fig. 2(b).

(2) $B$ regime; the tunnel motion follows the $B$ branch (see line $B$ in Fig. 1). The form of trajectory is presented in Fig. 2(a) ( $B$ curve). In this case the initial and the final points of the tunnel motion of the lattice are located on the $B$ branch (see Fig. 1, line $B$ ). To the left of the point $f$ of the line $B$ (see Fig. 1) the motion of the lattice will be in a classically permitted region and therefore will be in real time. Due to the classical motion along this part of the $B$ branch the lattice will occur on the $F$ branch, i.e., the exciton will be in the free state. Such classical motion along the $B$ and $F$ energy branches does not give an input to the imaginary part of the action. In all these cases the motion through the free $F$ branch is the classical one. Of course, generally speaking, it is also possible to have a tunnel motion through this branch. But this possibility is lost by adopting of the self model substitution.

(3) $A$ regime, which corresponds to the activational solution. The lattice does not move during the entire interval of the tunnel time $-\beta / 2<t<\beta / 2$. During the tunnel time it is located on the top of the self-trapped barrier (see Fig. 1, circle $A$ ). The form of trajectory is presented in Fig. 2(a) (see line $A$ ).

The mutual configuration of the energy branches, which depends on the deficiency of the photon energy $\Delta$ (see Fig. 1) and the temperature $T$, permits the existence of one of these regimes. Thus, for values of the deficiency energy $\Delta<\frac{1}{4}$ (note that the condition $\Delta<\frac{1}{4}$ does not contradict the condition $\Delta \gg \omega$, because the deficiency $\Delta$ is measured in units $W$ which is the value of the selftrapped barrier, but $W \gg \omega)$ only one $E B$ regime exists, since only one $E B$ class of trajectories is possible. For these trajectories the action may be explicitly calculated employing the formula

$$
\begin{aligned}
S(\beta, \Delta)=C_{0} & {\left[\int_{\tau}^{-\beta / 2}+\int_{\beta / 2}^{-\tau}\right]\left(\dot{\varphi}^{2}+\varphi^{2}-\varphi^{4}+\Delta\right) d t } \\
& \left.+\int_{-\tau}^{\tau}\left(\dot{\varphi}^{2}+\varphi^{2}\right) d t\right] .
\end{aligned}
$$

The tunnel motion begins at time $t=0$. At this time the lattice begins to tunnel over the $E$ branch. At the moment $t=\tau$ the optical transition occurs and the lattice transfers from the empty to the bound branch. In the intervals $-\beta / 2<t<\tau$ and $-\tau<t<\beta / 2$ the lattice moves along the bound branch. The point $\tau$ [the cuspoidal point, see Fig. 2(b)] is the point of the generation of an exciton in the bound state. The value of $\tau$ is determined from the condition that at the time moment $\tau$ the boundexciton energy is equal to the photon energy deficiency. The calculation gives

$$
\tau=\operatorname{arccosh}\left[\left(\Delta / \varepsilon^{2}\right)^{1 / 4}\right] .
$$

The quantity $\varepsilon$ is an energy parameter, which is related to the temperature of the lattice by the formula (the position of the horizontal line in Fig. 1 depends on the value $\varepsilon$ )

$$
\beta=-2 \tau+2 F\left(\varphi_{0}(x), k\right) / p
$$

in which $F(\varphi, k)$ is the partial elliptic integral of the first order:

$$
\begin{aligned}
& F(\varphi, k)=\int_{0}^{\varphi}\left(1-k^{2} \sin ^{2} t\right)^{-1} d t \\
& \varphi_{0}(x)=\arcsin \left\{p / x\left[\left(x^{2}-q^{2}\right) /\left(p^{2}-q^{2}\right)\right]^{-1 / 2}\right\}, \\
& x=\Delta^{1 / 4}, \quad k^{2}=\left(p^{2}-q^{2}\right) / p^{2},
\end{aligned}
$$

where

$$
\begin{aligned}
& p^{2}=\left\{1+[1-4(\varepsilon-\Delta)]^{1 / 2}\right\} / 2, \\
& q^{2}=\left\{1-[1-4(\varepsilon-\Delta)]^{1 / 2}\right\} / 2 .
\end{aligned}
$$

Solving the Lagrange equation for $\varphi(t)$ and calculating the action we obtain the following explicit expression: 


$$
\begin{aligned}
S(\beta, \Delta)=C_{0}[ & -\varepsilon \sinh 2 \tau+2 \varepsilon F\left(\varphi_{0}(x), k\right) / p \\
& +4\left\{(p / 3)\left[\left(p^{2}+q^{2}\right) E\left(\varphi_{0}(x), k\right)-2 q^{2} F\left(\varphi_{0}(x), k\right)\right]+\left\{\left(x^{2}-p^{2}-q^{2}\right)\left[\left(p^{2}-x^{2}\right)\left(x^{2}-q^{2}\right)\right]^{1 / 2} / 3 x\right\}\right.
\end{aligned}
$$

For details, see Ref. 6. In Eq. (12) the function $E(\varphi, k)$ is the partial elliptic integral of second order with amplitude $\varphi$ and modulus $k$, respectively. Here we have $\Delta \leq \varepsilon \leq \Delta^{1 / 2}$.

For deficiency energies $\Delta$ with $\frac{1}{4}<\Delta<1$ three regimes may exist. The condition of the existence of each regime depends on the temperature $\beta^{-1}$. At low temperature $\beta \gg 1$ we have the $E B$ regime. If the temperature is increased the $B$ and the $A$ regimes may exist. For the $B$ regime the action is determined by the formula

$$
S(\beta, \Delta)=C_{0}\left[\int_{-\beta / 2}^{\beta / 2}\left(\dot{\varphi}^{2}+\varphi^{2}-\varphi^{4}+\Delta\right) d t\right],
$$

where the initial and the final points of the tunnel motion are equal to the inverse temperature $\beta$ :

$$
\beta=2 F(\pi / 2, k) / p .
$$

Solving the Lagrange equation and using the formula (13) one can easily obtain the following expression for the action:

$$
\begin{aligned}
S(\beta, \Delta)=C_{0}\{ & 2(\varepsilon-\Delta) \\
+4 p(\pi / 2, k) / p & {\left[\left(p^{2}+q^{2}\right) E(\pi / 2, k)\right.} \\
& \left.\left.-2 q^{2} F(\pi / 2, k)\right]\right\},
\end{aligned}
$$

with $\Delta^{1 / 2} \leq \varepsilon \leq \Delta+\frac{1}{4}$.

The $A$ regime corresponds to the simple expression for the action:

$$
S(\beta, \Delta)=C_{0}\left(\frac{1}{4}+\Delta\right) \beta .
$$

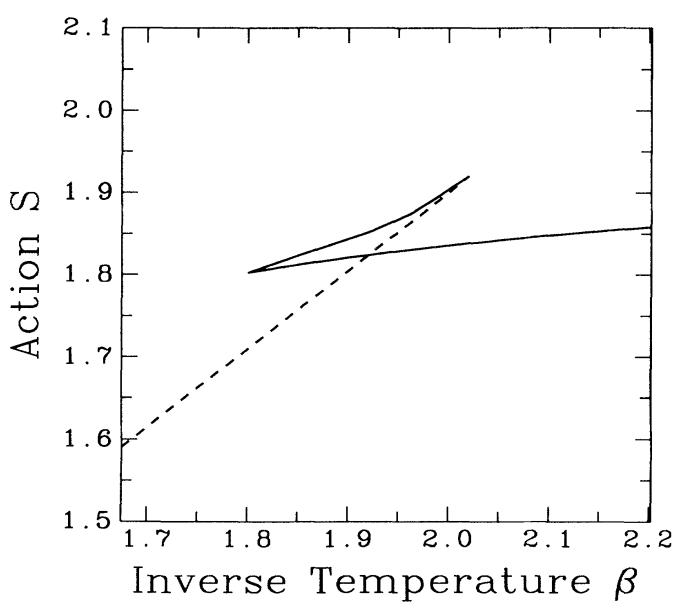

FIG. 3. The dependence of the action $S$ on the inverse temperature $\beta$ in the region of the first-order transition. The dashed line corresponds to an $A$ instanton. The solid line corresponds to an $E B F$ instanton.
At values of the deficiency $\Delta \geq 1$ the configuration of energy branches has a form such that only the $B$ and the $A$ regime can exist. The region of the existence of the regime may be evaluated with the aid of Eqs. (15), (14), and (12). Transitions from the $E B$ regime to the $A$ regime and from the $E B$ to the $B$ regime are transitions of the first order. The behavior of the dependence of the action on the temperature in the region of the transition for the free channel is presented in Fig. 3. It is the transition between the $A$ regime (an $A$ instanton corresponds to the dashed line) and the $E B F$ regime (an EBF instanton corresponds to the solid line; in the reduced model this instanton coincides with the $E B$ instanton). One can see that at the point of the transition the changing of the slope is not so small and it might be possible that this changing of the slope will not disappear if we take into account the preexponential factor for the coefficient of the light absorption. The transition from the $A$ to the $B$ regime is a transition of second order, i.e., there are no singularities at this point. The "phase diagram" of the regimes for the free channel considered is presented in Fig. 4. The solid line in this figure corresponds to the

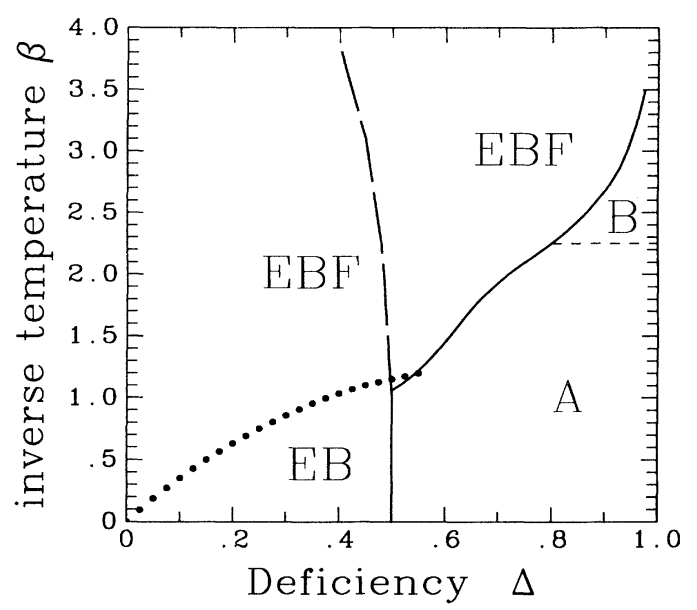

FIG. 4. The "phase" diagram of the existence of different regimes of the light absorption for the free channel. The letters $A, B, E B$, and $E B F$ designate the different regimes of the light absorption. The solid line corresponds to the transition between the regimes of the first order (the changing of the slope in the coefficient of the absorption). The dashed line corresponds to the smooth transition (the transition of the second order). On the long-dashed line the absorption in the free channel equals the absorption in the self-trapped channel. The dotted curve corresponds to the transition between the $E B$ and the $E B F$ regimes. 
transition of first order. The dashed line corresponds to the transition of second order.

In the self-trapped channel our reduced model describes also three regimes: the $E B$, the $B$, and the $A$ re- gime. The associated shapes of these trajectories are presented in Fig. 2(a), respectively.

For the $E B$ regime the expression for the imaginary part of the quasiclassical action has the form

$$
\begin{aligned}
S(\beta, \Delta)=C_{0}[ & -\varepsilon \sinh 2 \tau+(4 \Delta-2 \varepsilon) F\left(\varphi_{1}(x), k\right) / p \\
& \left.+4\left\{(p / 3)\left[\left(p^{2}+q^{2}\right) E\left(\varphi_{1}(x), k\right)-2 q^{2} F\left(\varphi_{1}(x), k\right)\right]-\left[\left(-x^{2}+p^{2}\right)\left(x^{2}-q^{2}\right)\right]^{1 / 2}(x / 3)\right\}\right],
\end{aligned}
$$

where $\Delta \leq \varepsilon \leq \Delta^{1 / 2}$,

$$
\varphi_{1}(x)=\arcsin \left\{\left[\left(-x^{2}+p^{2}\right) /\left(p^{2}-q^{2}\right)\right]^{1 / 2}\right\}, \quad \beta=2 \tau+2 F\left(\varphi_{1}(x), k\right) / p .
$$

In contrast, at values $0 \leq \varepsilon \leq \Delta$ the action corresponding to the same regime has a different form:

$$
\begin{aligned}
S(\beta, \Delta)=C_{0}[ & \varepsilon \sinh 2 \tau+(2 \varepsilon) F\left(\varphi_{2}(x), k_{2}\right) /\left(p^{2}-q^{2}\right)^{1 / 2} \\
+ & \left.4\left\{\left[\left(p^{2}-q^{2}\right)^{1 / 2} / 3\right]\left[2\left(p^{2}+q^{2}\right) E\left(\varphi_{2}(x), k_{2}\right)-q^{2} F\left(\varphi_{2}(x), k_{2}\right)\right]+\left[\left(-x^{2}+p^{2}\right)\left(x^{2}-q^{2}\right)\right]^{1 / 2}(x / 3)\right\}\right]
\end{aligned}
$$

where $\varphi_{2}(x)=\arccos (x / p), k_{2}=\left[p^{2} /\left(p^{2}-q^{2}\right)\right]^{1 / 2}$, and

$$
\beta=2 \operatorname{arccosh}(\Delta / \varepsilon)^{1 / 2}+F\left(\varphi_{2}(x), k_{2}\right) .
$$

For the $B$ regime the imaginary part of the quasiclassical action has the form

$$
\begin{aligned}
& S(\beta, \Delta)=C_{0}\{(2 \varepsilon) F(\pi / 2, k) / p \\
& +4 p / 3\left[\left(p^{2}+q^{2}\right) E(\pi / 2, k)\right. \\
& \left.\left.-2 q^{2} F(\pi / 2, k)\right]\right\}
\end{aligned}
$$

and the temperature $\beta$,

$$
\beta=2 F(\pi / 2, k) / p .
$$

For the $A$ regime of the self-trapped channel the action is

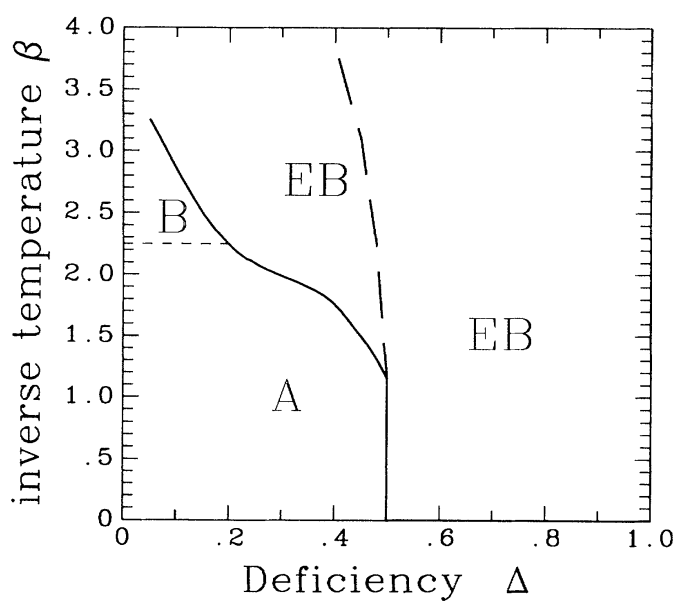

FIG. 5. The "phase" diagram of the existence of different regimes of the light absorption for the self-trapped channel (see caption of Fig. 4). given by expression (15).

The "phase" diagram of the existence of these regimes is presented in Fig. 5. The character of the transitions between the regimes is just the same as for the free channel (see, for comparison, Fig. 4).

\section{INSTANTONS AT SMALL VALUES OF PHOTON ENERGY DEFICIENCY}

The procedure of Sec. IV yields only three classes of trajectories of the free type. But according to the general classification of the free channel (see Ref. 1) there are five different classes of trajectories. Thus the $E B F$ and $B F$ regimes have been missed.

An additional class of trajectories of the free type $E B F$ class, see the form of trajectory in Fig. 2(b)] can be found by the method of the weakly bound state. ${ }^{7}$ This method can be applied because at values $\Delta<<1$ (but $\Delta \gg \omega / W$ ) the bound state of the exciton, corresponding to a free channel, is a weakly bound state. For these states the following formulas may be derived (for details, see Ref. 7): (1) for the phonon function $X(r, t)$ we get

$$
X(r, t)=\varphi(t) Q(r),
$$

and (2) for the wave function of the exciton we find

$\Psi(r, t)=\chi(r)\left(\exp \left\{-[2 E(t)]^{1 / 2} r\right\} / r\right)\left[E(t) /\left(2 \pi^{2}\right)\right]^{1 / 4}$.

Here $E(t)$ is an eigenvalue of the Schrödinger equation and the function $\chi(r)$ obeys the following boundary conditions:

$$
\chi(0)=0, \quad \chi(r)=\text { const at the value } r \rightarrow \infty .
$$

In the zeroth-order approximation for $\Delta<<1$ the Schrödinger equation yields a connection between the functions $\chi(r)$ and $Q(r)$ :

$$
\chi^{\prime \prime}-\chi Q=0 \text {. }
$$


Here we have used unit normalization, $\varphi(\tau)=1$. The energy of the bound state of the exciton is equal to (see Ref. 7):

$$
E(t)=B[\varphi(t)-1]^{2},
$$

where

$$
\begin{aligned}
& B=W_{0}^{2} / E_{0}, \\
& W_{0}=\int_{0}^{\infty} \chi^{\prime 2} / 4 d r, \\
& E_{0}=2 \pi^{2} \lim \chi(r) \text { at } r \rightarrow \infty .
\end{aligned}
$$

Using Eqs. (25) and (26) one may obtain the Lagrange function for the empty $E$, bound $B$, and free $F$ branches, respectively:

$$
\begin{aligned}
& L_{E}=A\left(\dot{\varphi}^{2}-\varphi^{2}\right), \\
& L_{B}=A\left(\dot{\varphi}^{2}-\varphi^{2}\right)-B(\varphi-1)^{2}-\Delta, \\
& L_{F}=A\left(\dot{\varphi}^{2}-\varphi^{2}\right)-\Delta,
\end{aligned}
$$

where $A=\int\left[\left(\chi^{\prime} / 2 \chi\right)^{2} / 2\right] d^{3} r$.

A model which is completely analogous to expression (27) has been solved in Ref. 1. Here, however, we must find the unknown function $\chi(r)$ at each value of the deficiency $\Delta$ and the temperature $T$. In other words, the curvature of the model obtained (27) depends on the function $\chi(r)$, i.e., it depends on quantities $\Delta$ and $T$. Here we solve this model by an iteration-variation procedure, using a trial function for $\chi(r)$ : $\chi(r)=r C /\left(D^{2}+r^{2}\right)^{1 / 2}$, where $C$ and $D$ are variational parameters. The details will be given elsewhere. ${ }^{6}$ In the result we have calculated the line of the transition between the $E B$ and $E B F$ regimes. It is presented by the dotted curve in Fig. 4. Thus, by this method, we have been able to determine the region of existence of the $E B F$ regime of light absorption in the free channel (see Fig. 2). The transition between the $E B F$ and the $E B$ regimes is of second order at small values of the deficiency. With increasing values $\Delta$ it may turn into a first-order transition (see Ref. 1 for comparison).

The region of the existence of the $B F$ regime cannot be determined by the method of the weakly bound state employed here. The region of the existence of the $B F$ instanton ( $B F$ solution) is located in the region of the $B$ regime in Fig. 4. This region corresponds to the photon energy deficiency, which is about one unit (see Fig. 4). But at these deficiency values the method of the weakly bound state is not applicable.

\section{RESULTS}

The phase diagrams obtained and the explicit expressions for the coefficient of the absorption of the light (expressions for the action) are the main results of the work presented here. The free channel of light absorption corresponds to the phase diagram presented in Fig. 1. There are four regions on this figure $(E B F, E B, B$, and $A)$. Each of them corresponds to a particular regime of the light absorption. There are two characters of the transition between regimes: the first order (see Fig. 3) and the second order (see Ref. 1). In the first case there is a singularity in the coefficient of the light absorption (the abrupt changing of the slope, see the transition from the dashed to the solid line in Fig. 3). In the second case the change in the coefficient of the light absorption is smooth. The solid line in Figs. 4 and 5 corresponds to the transition of first order, the dashed line corresponds to the transition of second order. In Fig. 5 one can see the regions of the regimes of light absorption in the selftrapped channel. There are three different regimes of light absorption ( $A, B$, and $E B$ ). The transition between the $A$ and $B$ regimes is of second order, other transitions are of first order. The boundary of the dominant region of the absorption for the free and self-trapped channel is indicated by the long-dashed lines in Figs. 4 and 5. The dominant region of the free channel corresponds to small deficiencies ( $E B$ and $E B F$ regimes, see Fig. 4). For these two regimes the trajectories have an inverse part of the motion [see cuspoidal respective trajectories presented in Fig. 2(b)]. The respective trajectories have a simple physical interpretation, i.e., during the tunnel motion the lattice goes through the same configuration of the deformation over the different energy branches with and without the exciton. On the other hand, for perspective trajectories [see Fig. 2(a)] the deformations of the lattice during the tunnel motion over the different energy branches are different. The study of the role of respective trajectories has not been taken into account in previous works, studying the light absorption at small photon energy deficiencies.

\section{DISCUSSION AND EXPERIMENT}

At any value of the deficiency energy $\Delta$ of the photon and temperature $T$ the self-trapped excitons as well as the free excitons may be generated, that is, there is the light absorption in both channels. At small values of the photon energy deficiency the number of created free excitons will be much larger than the number of generated selftrapped excitons, or, equivalently, the absorption in the free channel is larger than the absorption in the selftrapped channel. On the other hand, at large values of $\Delta$ the absorption in the self-trapped channel will be dominant. If the number of the created free excitons is equal to the number of generated self-trapped excitons, the light absorption in both channels is equal to each other. Thus the line where this equality holds is the boundary for each dominant region. It is presented by the longdashed curves in Figs. 4 and 5. Presumably, this boundary may not be observed in an experiment. On the other hand, in an experiment, singularities in the coefficient of the absorption, corresponding to the first-order transitions between the regimes (see the solid line on the "phase" diagrams) may be detected. Their singularities are very difficult to observe because of dispersive effects, which is due to the preexponential factor and the absorption of light in the parallel channel.

High-precision measurements of the absorption of light may be of some help. We expect that it will be possible in some cases of measuring the hot luminescence of excitons to determine the light absorption independently in each channel. 
Why is it important to separate the light absorption into two channels? The consequences of the absorption are different for each channel. The generation of the self-trapped excitons causes the production of point defects ${ }^{12-15}$ and reabsorption of atoms from the crystals ${ }^{16,17}$ due to the difference in energy for the selftrapped and free excitons. Therefore the number of point defects is proportional to the number of the self-trapped excitons. Measuring the concentration of the defects after the exciton production for a given photon energy deficiency value we may estimate at least approximately the number of generated self-trapped excitons.

In the self-trapped channel the dominant region corresponds to large values of the photon energy deficiency (see Fig. 5). However, it is interesting to note that due to these two different channels of light absorption there are two different parts of the absorption, which correspond to the different exponential functions. The total absorption is given by the sum of these functions. Therefore, to compare the absorption in the two different channels, we should take into account the preexponential factors. It may be also possible in experiments, where the total absorption is measured that the Urbach rule is due to nondominant regimes $A$ and $B$ with large preexponential factors. According to our results (14), (15), and (20) these regimes are exactly described by the Urbach rule.
It may be also possible to measure the absorption for the free channel. It depends on values of the exciton lifetime $\tau_{e}$ in the free state and the time $\tau_{s}$ of the selftrapping, which depends on the height of the self-trapped barrier $W$. If $\tau_{e}<<\tau_{s}$ the exciton generated in the free state will also radiate from the free state. Therefore, in this case by simultaneous measurements of the absorption and of the luminescence of light of free excitons one may estimate that part of the absorption that corresponds to the free channel. In the opposite limit $\tau_{e} \gg \tau_{s}$ the exciton cannot luminesce from the free state, because it will be quickly self-trapped and therefore in this case it is impossible to measure the partial absorption corresponding to the free channel. For other purposes, which are not related to the absorption of light, experiments of this type have been performed in rare-gas solids. ${ }^{14-17}$ Light absorption measurements should be done in the near future.

\section{ACKNOWLEDGMENTS}

I wish to thank S. V. Meshkov and E. I. Rashba for fruitful discussions. I acknowledge the kind hospitality of M. L. Ristig at the University of Cologne. This work was supported by the Alexander von Humboldt Foundation.
${ }^{1}$ F. V. Kusmartsev and S. V. Meschkov, Zh. Eksp. Teor. Fiz. 94, 250 (1988) [Sov. Phys. - JETP 67, 2098 (1988)].

2 J. D. Dow and D. Redfield, Phys. Rev. B 5, 594 (1972).

${ }^{3}$ E. I. Rashba and S. V. Iordanski, Zh. Eksp. Teor. Fiz. 74, 1979 (1978) [Sov. Phys. -JETP 47, 1030 (1982)].

${ }^{4}$ A. S. Ioselevitsch, Zh. Eksp. Teor. Fiz. 83, 743 (1982) [Sov. Phys. -JETP 56, 1297 (1982)].

${ }^{5}$ E. I. Rashba, in Excitons, edited by E. I. Rashba and M. D. Sturge (North-Holland, Amsterdam, 1982), p. 543.

${ }^{6}$ F. V. Kusmartsev, Zh. Eksp. Teor. Fiz. (Sov. Phys. - JETP) (to be published).

${ }^{7}$ F. V. Kusmartsev and S. V. Meschkov, Zh. Eksp. Teor. Fiz. 85, 1500 (1983) [Sov. Phys. - JETP 58, 870 (1983)].

${ }^{8}$ D. L. Dexter, Nuovo Cimento Suppl. 7, 245 (1958).

${ }^{9}$ Y. Toyozawa, Prog. Theor. Phys. 22, 445 (1959); 27, 89 (1962).

${ }^{10}$ M. Schreiber and Y. Toyozawa, J. Phys. Soc. Jpn. 51, 1528
(1982); 51, 1537 (1982); 51, 1544 (1982); 52, 318 (1980); 52, 1924 (1983); 53, 864 (1984).

${ }^{11}$ M. Schreiber, Phys. Rev. B 34, 2914 (1986).

${ }^{12}$ F. V. Kusmartsev, Fiz. Nizk. Temp. 6, 1046 (1980) [Sov. J.Low. Temp. Phys. 6, 509 (1980)].

${ }^{13}$ F. V. Kusmartsev and E. I. Rashba, Czech. J. Phys. B 32, 54 (1982).

${ }^{14}$ E. I. Savchenko, U. I. Ribalko, and I. J. Fugol, Pis'ma Zh. Eksp. Teor. Fiz. 41, 210 (1985) [JETP Lett. 41, 256 (1985)].

${ }^{15}$ I. J. Fugol, E. I. Savchenko, and D. N. Grigorashenko, in Modern Problems of Solid State Physics and Biophysics (Nauka Dumka, Kiev, 1982), p. 224.

${ }^{16}$ T. Kloiber, W. Laash, G. Zimmerer, F. Coletti, and J. M. Debever, Europhys. Lett. 7, 77 (1988).

${ }^{17}$ T. Kloiber and G. Zimmerer, Radiat. Eff. Defects Solids (U.K.) 109, 219 (1989). 